\title{
Retroperitoneal bronchogenic cyst: a rare incidentaloma discovered in a juvenile hypertensive patient
}

\author{
Hypertension Research (2014) 37, 595-597; doi:10.1038/hr.2014.38; published online 6 March 2014
}

Bronchogenic cysts are incidentally discovered by radiological examination or during surgical procedures. The symptoms are usually related to a tracheobronchial pathway obstruction and/or secondary infection or hemorrhage. These cysts most commonly occur in the subcarinal and parahilar areas in the mediastinum. However, they have also been found in retroperitoneal locations. ${ }^{1,2}$ Here, we present an interesting case of juvenile hypertension accompanying a left adrenal incidentaloma, which was pathologically diagnosed as a bronchogenic cyst post surgery.

A 27-year-old hypertensive man was referred to our hospital for examination of a left adrenal tumor that was incidentally discovered by abdominal computed tomography (CT). Hypertension and obesity had been noted in annual checkups dating back 5 years. The patient showed moderate hypertension (140-149/70 to $86 \mathrm{~mm} \mathrm{Hg}$ ) and obesity (body mass index, 31.3 ), although he had no Cushingoid features, such as central obesity and skin lesions. The abdominal CT showed a $5-\mathrm{cm}$ circumscribed mass measuring 50 Hounsfield units (HU) in the left adrenal region (Figure 1a, upper), and the right adrenal gland was detected to be normal (Figure 1a, lower). An abdominal ultrasound showed a cystic pattern in the left adrenal region (Figure 1b). On magnetic resonance imaging (MRI), the adrenal tumor was detected as an ISO-intensified tumor by T1-weighted images (Figure 1c), in which no T2-hyperintense signal was observed (Figure 1d). A fat suppression and spectral attenuated inversion recovery (SPAIR) scan of the MRI studies showed little inclusion of adipose tissue in the tumor (Figure 1e).
In the laboratory examinations, the blood cell count, liver and renal functions, blood glucose, and related tumor markers, including carcinoembryonic antigen ((CEA), $1.90 \mathrm{ng} \mathrm{ml}^{-1}$ (normal, <5.0)), carbohydrate antigen 19-9 ((CA19-9), 6.9 $\mathrm{U} \mathrm{ml}^{-1}$ (normal, $<40)$ ), and neuron-specific enolase $\left(8.95 \mathrm{ng} \mathrm{ml}^{-1} \quad\right.$ (normal, <16.3)), were normal, although moderate hypokalemia $\left(3.8 \mathrm{mmoll}^{-1}\right)$ was concomitantly detected. The preoperative basal endocrine data were as follows: adrenocorticotropin, $27.6 \mathrm{pg} \mathrm{ml}^{-1}$ (normal, 7.2-63.3); cortisol, $14.0 \mu \mathrm{g} \mathrm{dl}^{-1}$ (normal, 8-25); plasma renin activity, $1.5 \mathrm{ng} \mathrm{ml}^{-1} \mathrm{~h}^{-1}$ (normal, 0.2-2.7); aldosterone, $10.5 \mathrm{ng} \mathrm{dl}^{-1}$ (normal, 3.6-24); dehydroepiandrosterone sulfate, $164 \mu_{\mathrm{g} \mathrm{dl}}^{-1}$ (normal, 138-519); adrenaline, $0.01 \mathrm{ng} \mathrm{ml}^{-1}$ (normal, $<0.1)$; noradrenaline, $\quad 0.22 \mathrm{ng} \mathrm{ml}^{-1}$ (normal, 0.1-0.5); and dopamine, $0.02 \mathrm{ng} \mathrm{ml}^{-1}$ (normal, <0.03). The urinary catecholamine levels were within normal ranges, including adrenaline $(10.7 \mu \mathrm{g}$ per day, normal, 3-41), noradrenaline $(108.8 \mu \mathrm{g}$ per day, normal, 31-160), metanephrine (0.13 mg per day, normal, 0.04-0.18), normetanephrine $(0.19 \mathrm{mg}$ per day, normal, 0.10.28 ) and vanillylmandelic acid $(4.1 \mathrm{mg}$ per day, normal, 1.5-4.3). Note that the urinary excretions of aldosterone $(8.9 \mu \mathrm{g}$ per day, normal, $0-7.5)$ and cortisol (127 $\mu \mathrm{g}$ per day, normal, 26-187) were slightly elevated. An adrenal scintigram using ${ }^{131}$ I-adosterol showed an approximately normal pattern of bilateral uptake in the adrenal regions; with ${ }^{131}$ I-metaiodobenzylguanidine, the scintigram showed no significant accumulation in a whole-body scan.

Laparoscopic left adrenalectomy was performed based on the size of the adrenal tumor $\left(54 \times 38 \mathrm{~mm}^{2}\right)$ and considering a latent neoplasm. The resected tissue showed a brownish and dark-gray cystic tumor (Figure 1f). Partly compressed normal adrenal tissue was attached to the outer surface of the tumor (Figure 1g). Microscopically, the cystic portion was partially lined by ciliated columnar epithelium (Figure $1 \mathrm{~h}$ ) resting on fibrous connective tissue and smooth muscle cells containing mucous glands and hyaline (Figure 1i). Pulmonary parenchyma or teratomatous components were not found in the tumor. The resected tumor was pathologically diagnosed as an extra-adrenal retroperitoneal bronchogenic cyst. Post surgery, glucocorticoid replacement therapy was not required. As shown in Figure 1j, the patient's systolic and diastolic blood pressure was readily normalized $(117 / 67 \mathrm{~mm} \mathrm{Hg})$ after the adrenalectomy, and the serum potassium level gradually normalized $\left(4.3 \mathrm{mmoll}^{-1}\right)$ within 3 weeks. After one postoperative year, both the serum cortisol $\left(12.0 \mu \mathrm{g} \mathrm{dl}^{-1}\right)$ and aldosterone $\left(8.5 \mathrm{ng} \mathrm{dl}^{-1}\right)$ levels were also decreased compared with their preoperative levels.

Bronchogenic cysts arise from the foregut as malformations when normal bronchial tubes are developmentally separated. Most bronchogenic cysts occur in the mediastinum region, and only a few cases have been reported in the retroperitoneum. ${ }^{1,2}$ There are no gender-related incidence differences, but the age range of onset (i.e., 40-70 years) is older than our patient. Retroperitoneal bronchogenic cysts are usually localized in the left adrenal region and the superior body of the pancreas. ${ }^{3}$ Most bronchogenic cysts are pathologically benign, although malignant forms have been reported due to characteristically increased serum levels of tumor markers, including CEA and CA19-9. 


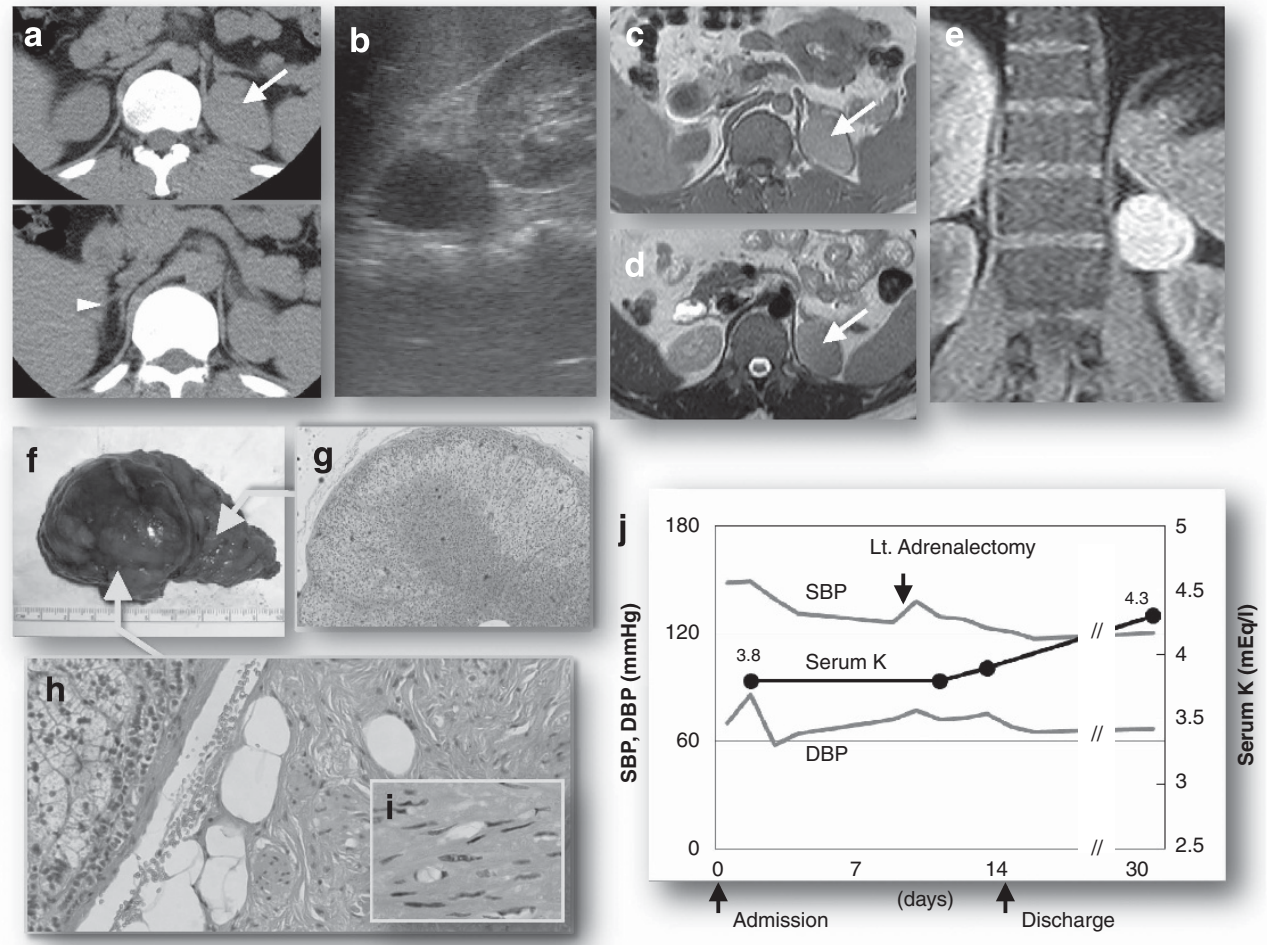

Figure 1 The radiological and pathological findings of the left adrenal tumor and the clinical course. (a) The left adrenal mass (indicated by an arrow) and right normal adrenal gland (indicated by an arrowhead) on plain CT. (b) An abdominal ultrasound. (c) A T1-weighted MRI (indicated by an arrow). (d) A T2weighted MRI (indicated by an arrow). (e) SPAIR-MRI. (f) The gross features of the resected adrenal tumor. (g) The histological findings of the adjacent adrenal tissue, hematoxylin and eosin (H\&E) staining $\times 40$. (h) The histological findings of the resected tumor, H\&E staining $\times 100$. (i) The microscopic findings of the resected tumor composed of bronchial-epithelial cells, H\&E staining $\times 400$. (j) Clinical course. SBP, systolic blood pressure; DBP, diastolic blood pressure; Serum K, serum potassium. A full color version of this figure is available at the Hypertension Research journal online.

In the present case, the tumor marker levels were within the normal ranges, and the imaging study and pathological workup confirmed that the resected adrenal tumor was benign.

The incidence of adrenal incidentalomas has been increasing because of recent advances in the availability of radiological devices. The CT attenuation value is informative for differentiating between benign and malignant adrenal tumors. A homogeneous mass with a smooth border and low HU value $(<10 \mathrm{HU})$ indicates a benign adenoma, except for lipid-poor adenomas. ${ }^{4} \mathrm{MRI}$ studies show benign adenomas as clear fat suppression of the signals by chemical-shift imaging, ${ }^{5}$ whereas adrenal metastasis is hypointense in T1-weighted images but hyperintense in T2-weighted images. Note that radiological examination occasionally uncovers various unexpected lesions that have originated from extra-adrenal tissues, namely adrenal pseudotumors, which may mislead the diagnosis of tumors. Adrenal pseudotumors, such as gastric diverticulum, splenic lobulation, renal, pancreatic or hepatic masses, and periadrenal varices, are found in $\sim 0.7 \%$ of incidentalomas, and the rate of preoperative discovery of such tumors is only $0.1 \% .^{6,7}$ Likewise, bronchogenic cysts are often misdiagnosed as solid adrenal tumors because of their rich protein contents. ${ }^{3,8}$ In the present case, the CT and MRI results indicated little inclusion of adipose tissue in the solid tumor, while ultrasound was informative and differentiated a cystic composition in the adrenal region.

The clinical hallmarks used as indicators to determine surgical intervention of adrenal incidentalomas are the initial size, growth rate and imaging characteristics of the adrenal tumor and the endocrine behavior.9 In the present case, the possibility of Cushing's syndrome, pheochromocytoma and primary aldosteronism was excluded preoperatively by endocrine data, including adrenal pressor hormones and adrenal scintigrams. However, considering the clinical course, the existence of the adrenal tumor may have been involved in the complication of hypertension. Doggett et al..$^{10}$ have reported a similar case of an adrenal bronchogenic cyst that was preoperatively considered to be pheochromocytoma. Despite the fact that the left adrenalectomy preparation was performed with an alpha-blocker (phenoxybenzamine), the authors reported that palpation during surgical exploration of the tumor immediately caused tachycardia and hypertension, possibly mediated by a catecholamine release because of an expanding mass or hemorrhage. ${ }^{10}$ Given that hypertension and moderate hypokalemia were normalized after surgery in our case, it is possible that a small amount of deviation of adrenocortical hormones contained in the tumor and/or those induced by the compressed normal adrenal was, at least somewhat, associated with the existence of preoperative hypertension.

Collectively, an accumulation of similar cases and pathophysiological investigations are needed in future studies. The present case further emphasizes the need to consider bronchogenic cysts as a differential diagnosis for left adrenal incidentaloma with juvenile hypertension.

\section{ACKNOWLEDGEMENTS}

We thank Dr Tetsuro Sei and Dr Nanako Ogawa for their constructive discussion regarding radiology. 
Tomohiro Terasaka ${ }^{1,2}$, Fumio Otsuka ${ }^{2}$, Kanako Ogura-Ochi ${ }^{1}$,

Tomoko Miyoshi ${ }^{1}$, Kenichi Inagaki ${ }^{1}$, Yasuyuki Kobayashi ${ }^{3}$, Yasutomo $\mathrm{Nasu}^{3}$ and

Hirofumi Makino ${ }^{1}$

${ }^{1}$ Department of Medicine and Clinical Science, Okayama University Graduate School of Medicine, Dentistry and Pharmaceutical Sciences, Kitaku, Okayama, Japan; ${ }^{2}$ Department of

General Medicine, Okayama University Graduate School of Medicine, Dentistry and

Pharmaceutical Sciences, Kitaku,

Okayama, Japan and ${ }^{3}$ Department of Urology, Okayama

University Graduate School of Medicine,
Dentistry and Pharmaceutical Sciences, Kitaku, Okayama, Japan

E-mail: fumiotsu@md.okayama-u.ac.jp

1 Govaerts K, Van Eyken P, Verswijvel G, Van der Speeten K. A bronchogenic cyst, presenting as a retroperitoneal cystic mass. Rare Tumors 2012; 4: e13.

2 O'Neal PB, Moore FD, Gawande A, Cho NL, King EE, Moalem J, Ruan D. Bronchogenic cyst masquerading as an adrenal tumor: a case of mistaken identity. Endocrine Pract 2012; 18: e102-e105.

3 Yang DM, Jung DH, Kim H, Kang JH, Kim SH, Kim JH, Hwang HY. Retroperitoneal cystic masses: CT, clinical, and pathologic findings and literature review. Radiographics 2004; 24: 1353-1365.

4 Kebapci M, Kaya T, Gurbuz E, Adapinar B, Kebapci N Demirustu C. Differentiation of adrenal adenomas (lipid rich and lipid poor) from nonadenomas by use of washout characteristics on delayed enhanced CT. Abdom Imaging 2003; 28: 709-715.

5 McDermott S, O'Connor OJ, Cronin CG, Blake MA. Radiological evaluation of adrenal incidentalomas: current methods and future prospects. Best Pract Res Clin Endocrinol Metab 2012; 26: 21-33.

6 Gokan T, Ohgiya Y, Nobusawa H, Munechika H. Commonly encountered adrenal pseudotumours on CT. Br J Radiol 2005; 78: 170-174.

7 Kodera R, Otsuka F, Inagaki K, Miyoshi T, Ogura T, Tanimoto $Y$, Sei T, Makino H. Gastric diverticulum simulating left adrenal incidentaloma in a hypertensive patient. Endocr J 2007; 54: 969-974.

8 Haddadin WJ, Reid R, Jindal RM. A retroperitoneal bronchogenic cyst: a rare cause of a mass in the adrenal region. J Clin Pathol 2001; 54: 801-802.

9 Aron D, Terzolo M, Cawood TJ. Adrenal incidentalomas. Best Pract Res Clin Endocrinol Metab 2012; 26: 69-82.

10 Doggett RS, Carty SE, Clarke MR. Retroperitoneal bronchogenic cyst masquerading clinically and radiologically as a phaeochromocytoma. Virchows Arch 1997; 431: 73-76. 